\title{
Multi-wavelength time domain diffuse optical tomography for breast cancer: initial results on silicone phantoms
}

Edoardo Ferocino, Giuseppe Di Sciacca, Laura Di Sieno, Alberto Dalla Mora, Antonio Pifferi, et al.

Edoardo Ferocino, Giuseppe Di Sciacca, Laura Di Sieno, Alberto Dalla Mora, Antonio Pifferi, Simon Arridge, Fabrizio Martelli, Paola Taroni, Andrea Farina, "Multi-wavelength time domain diffuse optical tomography for breast cancer: initial results on silicone phantoms," Proc. SPIE 10874, Optical Tomography and Spectroscopy of Tissue XIII, 108741N (1 March 2019); doi: 10.1117/12.2508657

SPIE. Event: SPIE BiOS, 2019, San Francisco, California, United States 


\title{
Multi-wavelength time domain Diffuse Optical Tomography for breast cancer: Initial results on silicone phantoms
}

\author{
Edoardo Ferocino $^{* a}$, Giuseppe Di Sciacca ${ }^{\mathrm{b}}$, Laura Di Sieno ${ }^{\mathrm{a}}$, Alberto Dalla Mora ${ }^{\mathrm{a}}$, Antonio Pifferi ${ }^{\mathrm{a}, \mathrm{d}}$, \\ Simon Arridge ${ }^{\mathrm{b}}$, Fabrizio Martelli ${ }^{\mathrm{c}}$, Paola Taroni ${ }^{\mathrm{a}, \mathrm{d}}$, Andrea Farina $^{\mathrm{d}}$ \\ ${ }^{a}$ Politecnico di Milano, Dipartimento di Fisica, Piazza Leonardo da Vinci, 32, 20133, Milan, Italy; \\ ${ }^{b}$ University College London, Department of Computer Science, Gower Street, London WC1E 6BT, \\ United Kingdom; \\ ${ }^{c}$ Università degli Studi di Firenze, Dipartimento di Fisica e Astronomia, Via G. Sansone 1, 50019 \\ Sesto Fiorentino, Firenze, Italy; \\ ${ }^{\mathrm{d}}$ Consiglio Nazionale delle Ricerche, Istituto di Fotonica e Nanotecnologie, Politecnico di Milano, \\ Piazza Leonardo da Vinci, 32, 20133, Milan, Italy
}

\begin{abstract}
Time domain Diffuse Optical Tomography (TD-DOT) non-invasively probes the optical proprieties of biological tissue. These can be related to changes in tissue composition, thus making TD-DOT potentially valuable for cancer imaging. In particular, an application of interest is therapy monitoring for breast cancer. Thus, we developed a software tool for multi-wavelength TD-DOT in reflectance geometry. While the use of multiple wavelengths probes the main components of the breast, the chosen geometry offers the advantage of linking the photon flight time to the investigated depth. We validated the tool on silicone phantoms embedding an absorbing inclusion to simulate a malignant lesion in breast tissue. Also, we exploited the a priori information on position and geometry of the inclusion by using a morphological prior constraint. The results show a good localization of the depth of inclusion but a reduced quantification. When the morphological constraint is used, though, the localization improves dramatically, also reducing surface artifacts and improving quantification as well. Still, there is room for improvement in the quantification of the "lesion" properties.
\end{abstract}

Keywords: Diffuse Optical Tomography, Time Domain, Reflectance, Breast cancer

\section{INTRODUCTION}

Tomography of tissues is a commonly used in medical imaging to provide diagnostic information. On the other hand, conventional tomographic imaging techniques (e.g., Computed Tomography or Magnetic Resonance Imaging) either employ ionizing radiation or complex magnetic fields analysis, having high costs for social care as well. These features make them not ideal for one important field of application: monitoring of neoadjuvant chemotherapy of breast cancer ${ }^{1}$, where repeated imaging sessions are required.

A valid technique that could support the established ones is Diffuse Optical Tomography (DOT): laser light in the NearInfrared (NIR) spectral region is injected in the tissue at several source locations arranged onto the tissue surface to form a specific pattern with the corresponding detectors. A wide volume of the tissue is probed, and the optical properties (absorption and reduced scattering coefficients $-\mu_{a}$ and $\mu_{s}^{\prime}$ respectively) can be retrieved ${ }^{2}$. When working with multiple wavelengths, it is possible to exploit the wavelength dependency of the optical properties of the tissue to correlate $\mu_{a}$ and $\mu_{s}^{\prime}$ to changes in the breast tissue composition and structure, and thus following the effects of neo-adjuvant chemotherapy $(\mathrm{NAC})^{3}$. If the laser sources are pulsed, the Time Domain (TD) approach is used to decouple absorption from scattering effects in a single measurement ${ }^{4}$. Moreover, the use of reflectance geometry exploits the link between photons arrival time and probed depth. TD-DOT is thus a non-invasive technique that can provide physiological information on the breast tissue composition and thus could be effectively employed in NAC monitoring ${ }^{5}$.

* edoardo.ferocino@polimi.it; phone +39 02 2399 6131; https://www.fisi.polimi.it/it/user/1511

Optical Tomography and Spectroscopy of Tissue XIII, edited by Sergio Fantini, Paola Taroni, Proc. of SPIE Vol. 10874, 108741N · C 2019 SPIE · CCC code: 1605-7422/19/\$18 · doi: 10.1117/12.2508657 
We developed a Matlab tool for multi-wavelength TD-DOT and we tested the reconstruction algorithm on silicone phantoms with an embedded localized heterogeneity, to simulate a breast lesion. Moreover, we exploited the information on the localization of the inclusion to increase spatial resolution of the TD-DOT. In the forecast of a clinical application of TD-DOT, information on localization of the breast lesion could be given by combination with ultrasound imaging.

\section{MATERIALS AND METHODS}

The TD-DOT approach that we implemented is composed by two main sections: a forward model describing photon propagation in the tissue and an inverse problem describing optical properties retrieval from a photon time distribution. The forward model is based on the analytical solution of the diffusion equation in a semi-infinite medium. When considering heterogeneities in the medium, a perturbative approach was used under the Born approximation after a discretization of the volume (i.e. voxelization) ${ }^{6}$.

To retrieve the optical properties from experimental time domain reflectance data, we solved the inverse problem by the following:

$$
\Delta \mu=\arg \min _{\Delta \mu}\left\{\sum_{i}\left(\frac{M_{i}-J_{i} \Delta \mu}{\sigma_{i}}\right)^{2}+\mathcal{R}(\Delta \mu)\right\}
$$

where $\Delta \mu$ is the vector of the perturbations in each voxel, $M_{i}$ is the measured photon-count for the $i$-th combination of sources and detectors, $J_{i}$ is the $i$-th row of the Jacobian, $\sigma_{i}=\sqrt{M_{i}}$ is the standard deviation accordingly to the Poisson statistics, and $\mathcal{R}$ is a regularization expression. The analytical expression of the Jacobian is taken from reference ${ }^{6}$ and convoluted with the Instrument Response Function.

To exploit the information on the localization of the heterogeneity referred as inclusion, we included in the reconstruction algorithm a morphological prior constraint. This acts as a soft constraint, preserving the edges of the inclusion and helping to improve spatial resolution ${ }^{7}$.

A validation of the overall approach was carried out on silicone phantoms employing 8 wavelengths $(620 \mathrm{~nm}, 670 \mathrm{~nm}$, $740 \mathrm{~nm}, 800 \mathrm{~nm}, 910 \mathrm{~nm}, 1020 \mathrm{~nm}, 1050 \mathrm{~nm}, 1090 \mathrm{~nm}$ ) selected in correspondence with characteristic spectral features of silicone absorption, showing slightly decreasing absorption for increasing wavelengths and sharp peaks around 910, 1020 and $1090 \mathrm{~nm}$. In the center of a silicone slab (100 mm width, $120 \mathrm{~mm}$ length, $40 \mathrm{~mm}$ thickness) of homogeneous material with known optical properties (nominal values: $\mu_{a}=0.005 \mathrm{~mm}^{-1}, \mu_{s}^{\prime}=1 \mathrm{~mm}^{-1}$ at $670 \mathrm{~nm}$ ), we placed an inclusion achieving a contrast for the absorption coefficient mimicking a strongly absorbing lesion (e.g. a tumor with high hemoglobin content). The inclusions we employed are cylinders of different size: i) $11 \mathrm{~mm}$ diameter and $10 \mathrm{~mm}$ height, center is set at $10 \mathrm{~mm}$-depth, ii) $22 \mathrm{~mm}$ diameter and $15 \mathrm{~mm}$ height, center is set at $22.5 \mathrm{~mm}$-depth. For each geometry, two values of contrast for the absorption were used: $\mu_{a}=0.01 \mathrm{~mm}^{-1}, \mu_{a}=0.02 \mathrm{~mm}^{-1}$ (nominal values at 670 $\mathrm{nm}$ ). Overall 4 combinations of phantoms were tested. We will discuss only one combination in detail: geometry $i$ ) with $\mu_{a}=0.02 \mathrm{~mm}^{-1}$.

Four measure locations were set - equally spaced - on each long side of a rectangular shape (40 $\mathrm{mm} \times 28 \mathrm{~mm})$, thus yielding overall 8 measure points. Measures were performed exploiting the possible combinations of sources and detectors one at a time, neglecting the configuration in which source and detector are co-located. Measures were made in reflectance geometry. The light source was a supercontinuum laser and wavelength selection was made by a rotating prism: an optical fiber collected light and was put in contact to the silicone phantom. The re-emitted light was then transferred by an optical fiber to a silicon photomultiplier (SiPM) ${ }^{8}$. A PC board for time-correlated single photon counting was used to acquire the time distributions of the reflected photons.

Table 1 shows the optical properties at all wavelengths of the phantom bulk and of the localized heterogeneity, as measured from wide blocks of the corresponding material.

The measured optical properties of the bulk were used as background values in the reconstruction algorithm of the perturbative approach. 
Table 1. Measured optical properties for the inclusion and the bulk

\begin{tabular}{rcc|cc}
\hline \multirow{2}{*}{$\begin{array}{c}\text { Wavelength } \\
(\mathrm{nm})\end{array}$} & \multicolumn{2}{c|}{$\mu_{s}^{\prime}\left(\mathrm{mm}^{-1}\right)$} & \multicolumn{2}{c|}{$\mu_{a}\left(\mathrm{~mm}^{-1}\right)$} \\
\cline { 2 - 5 } & Inclusion & Bulk & Inclusion & Bulk \\
\hline $\mathbf{6 2 0}$ & 0.988 & 1.213 & 0.0168 & 0.0052 \\
$\mathbf{6 7 0}$ & 0.930 & 1.082 & 0.0172 & 0.0047 \\
$\mathbf{7 4 0}$ & 0.920 & 0.950 & 0.0196 & 0.0058 \\
$\mathbf{8 0 0}$ & 0.799 & 0.840 & 0.0175 & 0.0046 \\
$\mathbf{9 1 0}$ & 0.599 & 0.655 & 0.0245 & 0.0102 \\
$\mathbf{1 0 2 0}$ & 0.475 & 0.583 & 0.0198 & 0.0104 \\
$\mathbf{1 0 5 0}$ & 0.458 & 0.531 & 0.0154 & 0.0054 \\
$\mathbf{1 0 9 0}$ & 0.415 & 0.515 & 0.0178 & 0.0092 \\
\hline
\end{tabular}

\section{RESULTS AND DISCUSSION}

We used two main figures of merit to assess the overall quality of the reconstruction: localization and quantification. Localization is quantified by the difference between the reconstructed center of mass of the inclusion and its actual position in the bulk. Quantification is measured by the error between the integral reconstructed optical value in a limited region of interest around the center of mass and the corresponding theoretical value.

Each of the following Figures shows either $\mu_{a}$ or $\mu_{s}^{\prime}$ reconstructed in the volume at $670 \mathrm{~nm}$. The $X$ axis of each subplot of a single Figure shows the $x$ coordinate in the volume, and the $Y$ axis the $y$ coordinate. Different subplots refer to different values of the $z$ coordinate or depth, from $z=0 \mathrm{~mm}$ to $z=30 \mathrm{~mm}$. In each dimension, the volume is discretized with $2 \mathrm{~mm}$ pace. The colorbar indicates the span of the reconstructed optical values in $\mathrm{mm}^{-1}$ units.

Figure 1 shows the reconstructed absorption coefficient. The reconstructed inclusion is located deeper than the actual one as the center of mass is set around $12 \mathrm{~mm}$, but the overall inclusion height is correctly reconstructed as the inclusion occupies around 5 subplots and thus $10 \mathrm{~mm}$. At the same time, for a fixed $z$, the image is blurred and spread along $x-y$ showing low localization in that plane. On the surface, artifacts can be seen especially at $z=0 \mathrm{~mm}$ and $z=2 \mathrm{~mm}$, resembling the sources and detectors pattern. The sign of the inclusion is properly reconstructed, but quantification is generally poor, with strong underestimation (relative error up to $70 \%$ ). 

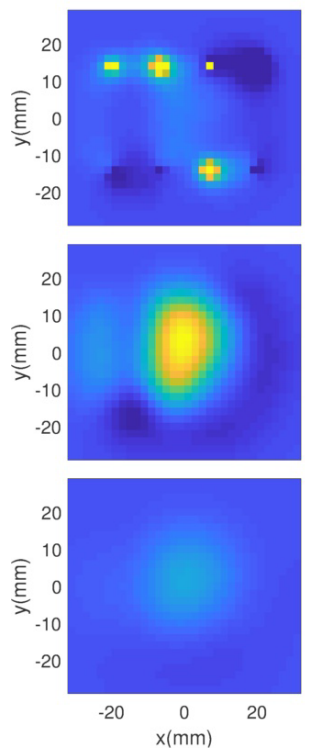
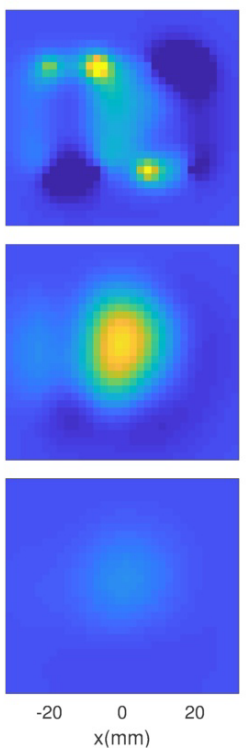
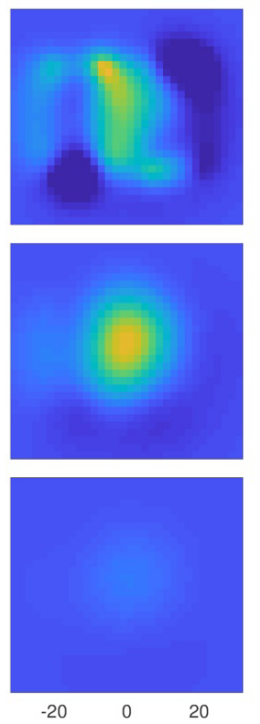
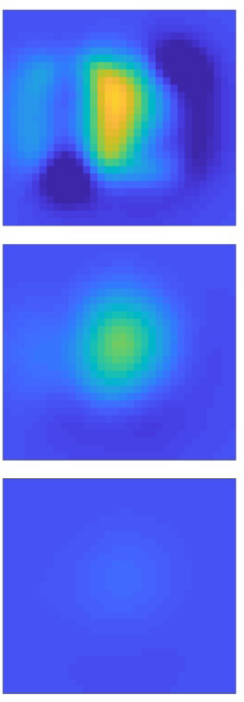

$-20 \quad 0 \quad 20$
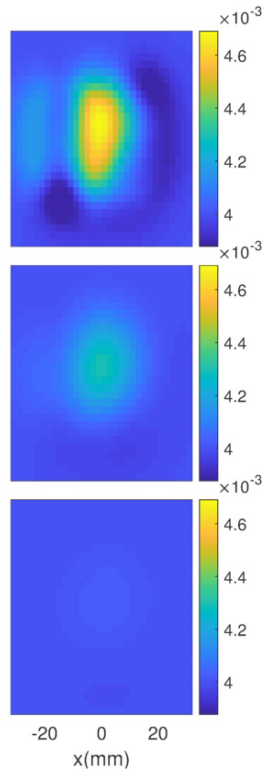

Figure 1. Reconstructed $\mu_{a}$ at $670 \mathrm{~nm}$. Good localization along the $z$ axis is reached, while quantification is poor.

The reconstructed reduced scattering coefficient is shown in Figure 2. The reconstruction is noisy and there are more artifacts on the surface respect to the absorption image (Figure 1). Also, the reconstructed inclusion is wide and more scattering respect to the background apparently in contrast with values in Table 1. Though, this behavior is expected as the nominal contrast of the inclusion respect to the background for the scattering parameter is zero (with measured values differing by $<15 \%$ ).
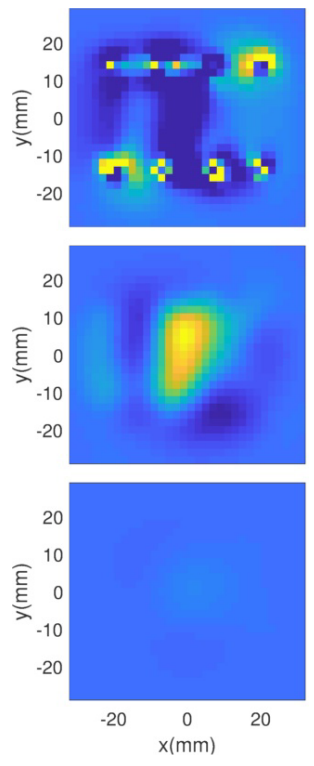
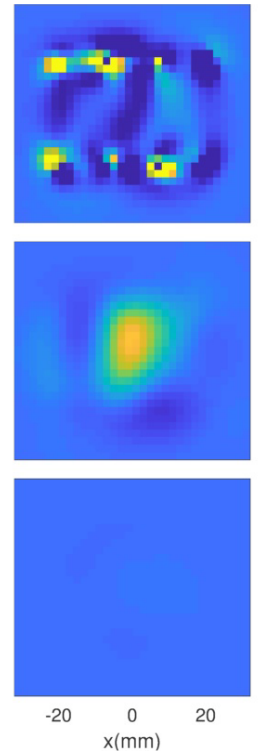
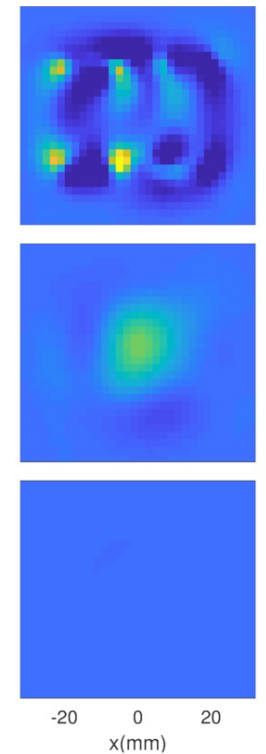
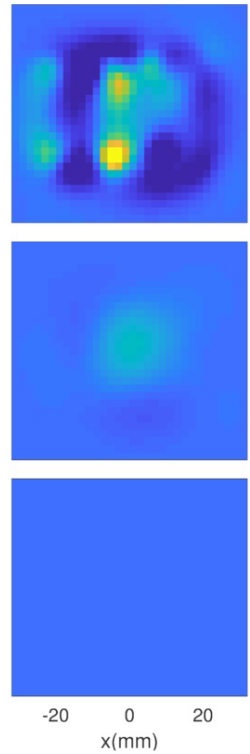
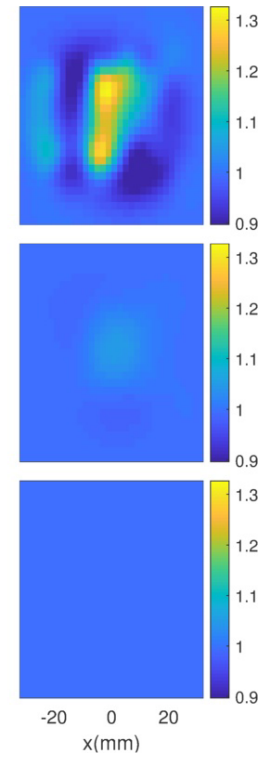

Figure 2. Reconstructed $\mu_{s}^{\prime}$ at $670 \mathrm{~nm}$. Reconstruction is noisy, due to low contrast $(<15 \%)$ of the scattering coefficient respect to the background.

An important parameter we tested is the effect of exploiting the a-priori information on the geometry and position of the inclusion. So, the Figures reported in the following were obtained with the use of the morphological prior constraint as explained in section "Materials and Methods". In particular, Figure 3 shows the reconstructed absorption coefficient. Comparing Figure 3 with Figure 1, a significant improvement in terms of localization can be seen. The top of the inclusion is correctly reconstructed at around $6 \mathrm{~mm}$ from the surface. At the same time, the inclusion is spread along the $z$ axis in 6 subplots thus having $12 \mathrm{~mm}$ height. The blurring in the $x-y$ plane disappears along with most of the artifacts of 
the surface, even though some negative rebounds are still present. The improvement in localization, especially the reduction of artifacts positively affects also quantification, as the maximum error is reduced to $50 \%$.
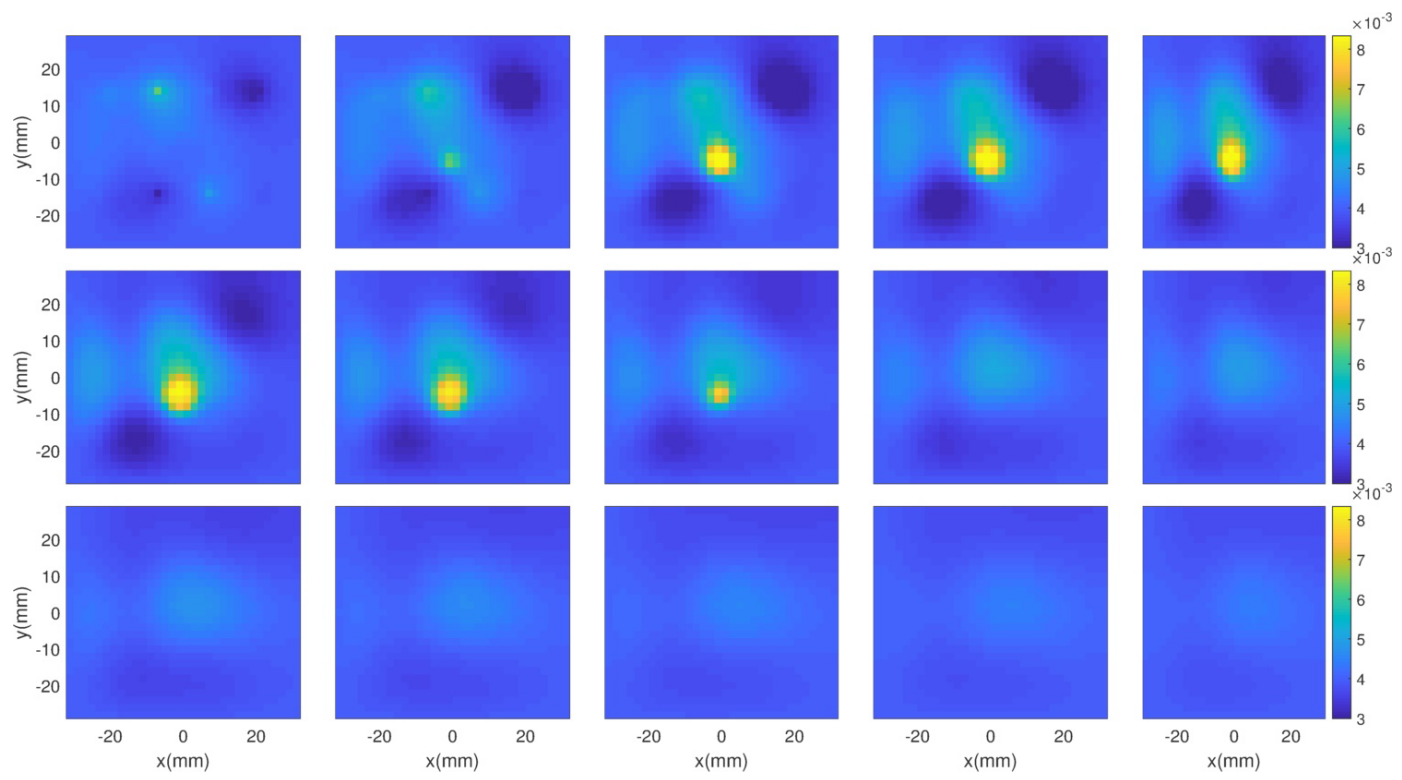

Figure 3. Reconstructed $\mu_{a}$ at $670 \mathrm{~nm}$ with the use of the morphological prior constraint. Localization strongly improves and quantification benefits as well.

The use of the morphological prior constraint affects also the scattering coefficient reconstruction as shown in Figure 4. The strong artifacts on the surface are dampened and the blurring of the inclusion is diminished to few pixels suggesting the presence of a small scattering perturbation. Though, quantification does not follow the right direction, as the contrast respect to the background increases as compared to Figure 2.
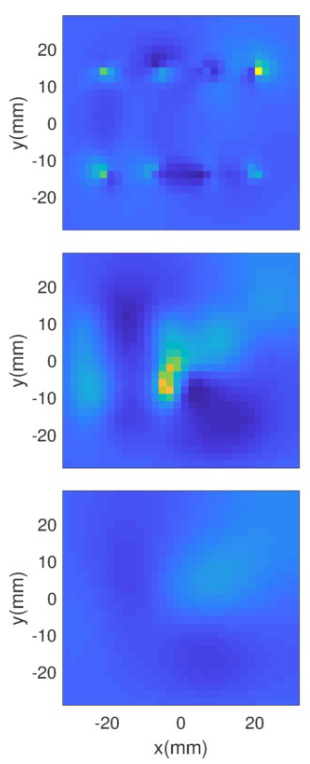
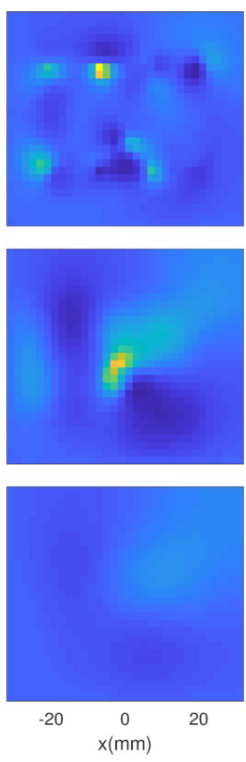
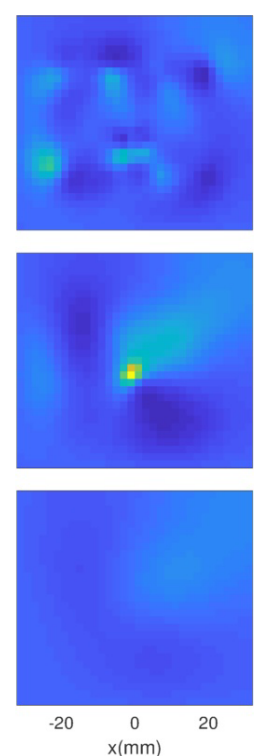
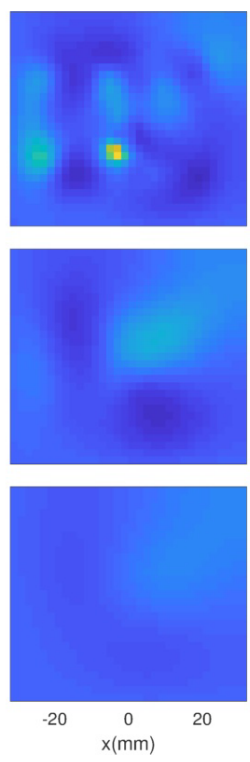
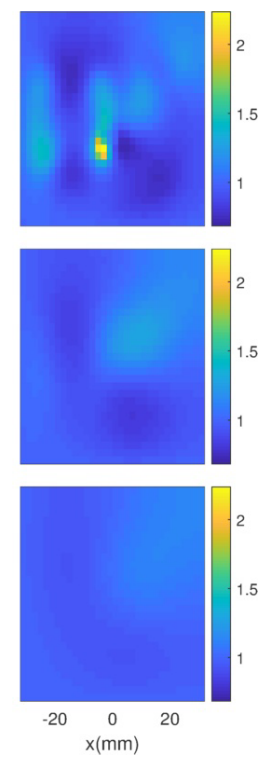

Figure 4. Reconstructed $\mu_{s}^{\prime}$ at $670 \mathrm{~nm}$ with the use of the morphological prior constraint. A small perturbation is reconstructed, but with marked error on quantification (high contrast of wrong sign respect to the background).

\section{CONCLUSIONS}

Effective therapy monitoring for breast cancer (and potentially even prediction of pathologic outcome) would be crucial to increase effectiveness and benefit the patient, while no conventional imaging technique allows it. Time domain 
Diffuse Optical Tomography could be a valid technique to probe the optical parameters of the tissue that correlate with tumor composition. Thus, we developed a Matlab tool for multi wavelength time domain Diffuse Optical Tomography of the breast. We tested it on silicone phantoms embedding a localized inhomogeneity, mimicking a lesion. A compact rectangular geometry was employed for the pattern of sources and detectors. Also, a morphological prior constraint was included in the reconstruction algorithm for the exploitation of the a priori information on position and geometry of the inclusion. The constraint was tested in view of information that in clinical settings could be obtained by ultrasound imaging.

We estimated the reconstructed optical properties (absorption and reduced scattering coefficients) of the whole volume. The absorption reconstruction allows good depth localization of the inclusion (error $<2 \mathrm{~mm}$ ) even though some blurring affects the in-plane localization. The scattering reconstruction shows some strong surface artifacts. The quantitative estimate of optical parameters is generally poor, but the correct sign of the inclusion is retrieved.

With the addition of the morphological constraint, localization strongly improves both in absorption and scattering. At the same time, the surface artifacts are removed and quantification is improved, at least for the optical parameter with high contrast, i.e. absorption. Though, the overall quantification error is still high.

The obtained results are encouraging, and next development might include the use of a Finite Element approach to overcome the limitations of the linear perturbative model employed in the present work, fostering an improvement in the quantification figure of merit.

\section{ACKNOWLEDGEMENTS}

The research leading to these results has received partial funding from the European Union's Horizon 2020 research and innovation program under project SOLUS: "Smart Optical Laser and Ultrasound Diagnostics of Breast Cancer" (www.solus-project.eu, grant agreement No 731877). The project is an initiative of the Photonics Public Private Partnership. The authors declare that there are no conflicts of interest related to this article.

\section{REFERENCES}

[1] Mieog, J. S. D., van der Hage, J. A., and van de Velde, C. J. H., "Neoadjuvant chemotherapy for operable breast cancer," Br. J. Surg. 94, 1189-1200 (2007).

[2] Durduran, T., Choe, R., Baker, W., and Yodh, A., "Diffuse optics for tissue monitoring and tomography," Reports Prog. Phys. 73, 076701 (2010).

[3] Grosenick, D., Rinneberg, H., Cubeddu, R., and Taroni, P., "Review of optical breast imaging and spectroscopy," J. Biomed. Opt. 21, 091311 (2016).

[4] Dalla Mora, A., Contini, D., Arridge, S., Martelli, F., Tosi, A., Boso, G., Farina, A., Durduran, T., Martinenghi, E., Torricelli, A., and Pifferi, A., "Towards next-generation time-domain diffuse optics for extreme depth penetration and sensitivity," Biomed. Opt. Express 6, 1749 (2015).

[5] Tromberg, B. J., Pogue, B. W., Paulsen, K. D., Yodh, A. G., Boas, D. A., and Cerussi, A. E., "Assessing the future of diffuse optical imaging technologies for breast cancer management," Med. Phys. 35, 2443-2451 (2008).

[6] Martelli, F., Del Bianco, S., Ismaelli, A., and Zaccanti, G., Light Propagation through Biological Tissue and Other Diffusive Media: Theory, Solutions, and Software (SPIE Press Monograph, 2009).

[7] Douiri, A., Schweiger, M., Riley, J., and Arridge, S., "Anisotropic diffusion regularization methods for diffuse optical tomography using edge prior information," Meas. Sci. Technol. 18, 87-95 (2007).

[8] Konugolu Venkata Sekar, S., Dalla Mora, A., Bargigia, I., Martinenghi, E., Lindner, C., Farzam, P., Pagliazzi, M., Durduran, T., Taroni, P., Pifferi, A., and Farina, A., "Broadband (600-1350 nm) Time-Resolved Diffuse Optical Spectrometer for Clinical Use," IEEE J. Sel. Top. Quantum Electron. 22, 7100609 (2016). 